\title{
Live and let die: epigenetic modifications of Survivin and Regucalcin in non-small cell lung cancer tissues contribute to malignancy
}

Dörte Nitschkowski ${ }^{1,2,3 \dagger}$, Sebastian Marwitz ${ }^{1,3 \dagger}$, Sousana A. Kotanidou ${ }^{1,3}$, Martin Reck ${ }^{2,3}$, Christian Kugler ${ }^{3}$, Klaus F. Rabe ${ }^{2,3}$, Ole Ammerpohl ${ }^{2,4,5+}$ and Torsten Goldmann ${ }^{1,3^{*}+}$ (D)

\begin{abstract}
Recently, it was shown that the epigenetic age of non-small cell lung cancer (NSCLC) tissues is different from the chronological age of patients. Here, we demonstrate that Regucalcin and Survivin, molecules which are known to be involved in the process of aging and overcoming aging, are epigenetically modified in NSCLC tissues compared to corresponding tumor-free tissues from the same donors by using methylome bead chip and corresponding transcriptome analyses. A high expression of Survivin on the RNA level was negatively correlated with patients' survival in adenocarcinomas while a high Regucalcin expression was correlated positively. In stage 1 adenocarcinomas, this separation is even sharper for both genes. Within these, adenocarcinomas, smokers with low expression of Survivin show a better outcome, while the high expression of Regucalcin seems to be protective in never smokers. On the protein level, these molecules were detected by immunohistochemistry using tissue microarrays. Since Survivin can be secreted and we observed a high abundance of the protein also in the adjacent immune cells of the tumor microenvironment, an effect on benign cells can be assumed. These findings show that epigenetic re-programming of Survivin and Regucalcin in non-small cell lung cancer leads to enhanced expression of Survivin and reduced expression of Regucalcin, with a possible role of both molecules as predictive markers.
\end{abstract}

Keywords: Survivin (BIRC5), Regucalcin (RGN), Transcriptome, Methylome, Non-small cell lung cancer (NSCLC), Proliferation, Senescence

\section{Main text}

The human life span is limited through a gradual loss of function which occurs in the organism at different levels. This process of aging leads to diseases such as osteoporosis, pulmonary insufficiency, and cancer [1]. Utilizing Horvath's clock, we recently showed that different epigenetic aging takes place in NSCLC entities and does not match the chronological age of the patients [2]. Two genes known to be centrally involved

\footnotetext{
* Correspondence: tgoldmann@fz-borstel.de

${ }^{+}$Ammerpohl $\mathrm{O}$ and Goldmann T shared senior authorship.

${ }^{1}$ Pathology of the University Medical Center Schleswig-Holstein, Campus Lübeck and the Research Center Borstel, Leibniz Lung Center, Borstel, Germany

${ }^{3}$ Airway Research Center North (ARCN), Member of the German Center for Lung Research (DZL), Großhansdorf, Germany

Full list of author information is available at the end of the article
}

in proliferation and senescence processes, which are not considered in Horvath's algorithm, are Survivin $(B I R C 5)$ and Regucalcin (RGN). BIRC5 is known as a dual functional regulator that inhibits apoptosis and promotes proliferation with a negative effect on survival $[3,4]$, while $R G N$ is associated with aging and positively contributes to the outcome of cancer patients [5].

Therefore, we investigated epigenetic modifications of the BIRC5 and RGN genes in 33 patients (20 males, 13 females) by Infinium HumanMethylation450k BeadChips (Illumia Inc.) as previously described. All tumors were primary NSCLC and comprised 15 adenocarcinomas and 18 squamous cell carcinomas of the lung; the mean patient age at surgery was 65.6 years. Of these 33 patients, 18 were 
analyzed by transcriptome analysis as recently shown [6]. Relative quantile-normalized gene expression values for BIRC5 and RGN were extracted from the GEO-dataset GSE74706 and analyzed with GraphPad Prism v.6 (Fig. 1b). Protein level expression was determined by immunohistochemistry (IHC) utilizing polyclonal antibodies (BIRC5: Thermo Fischer Scientific, 1:50 dilution; RGN: Antibodies-online.com, 1:100 dilution) and ZytoChem-Plus-HRP with aminoethylcarbazole as a chromogen (Zytomed Systems) on tissue microarrays of formalin-fixed NSCLC tissues from 40 cases (21 adenocarcinomas, 19 squamous cell carcinomas) and matched controls (Fig. 1d). Hierarchical clustering of methylation levels of BIRC5 and RGN CpG loci obtained by GenomeStudio software was visualized by OMICS Explorer 2.1 (Qlucore). For validating the outcome of the DNA methylation data analysis, additional methylation data of 919 cases (412 squamous cell carcinomas and 507 adenocarcinomas, including controls) provided by the TCGA consortium was accessed from the GDC data portal (https:// portal.gdc.cancer.gov/).

Regarding the methylation of BIRC5 and RGN, we observed a clear differentiation of tumor-free and lung cancer tissues (Fig. 1a). Loci located in the downstream region of the BIRC5 gene (3'UTR, gene body; according to the information provided by the manufacturer) were hypermethylated in the tumor samples as compared to the controls whereas loci located in the transcription start site (TSS) and CpG islands were not aberrantly methylated in our analysis. In contrast, CpG loci of the $R G N$ gene located in CpG islands and at the transcription start site were hypermethylated in tumor samples. This outcome is in line [7] with an unambiguous increase in RNA transcription levels of BIRC5 in tumors as compared to corresponding tumor-free tissues and clearly decreased transcription levels of $R G N$ (Fig. 1b). In tumor samples, DNA hypermethylation of the $6 \mathrm{CpG}$ loci located in the 3'UTR of BIRC5 correlated significantly with an increase in BIRC5 expression (Pearson correlation coefficient (coeff) 0.515 to $0.633, p$ value 0.0013 to $3.442 \times 10^{-5}$ ), while hypermethylation of loci located in the TSS200 and 5'UTR of RGN correlated inversely with the expression of RGN (cg03139204 coeff $-0.373, p$ value 0.0249 ; cg06472116 coeff $0.363, p$ value 0.0298 ; cg19639792 coeff $-0.454, p$ value 0.0054$)$. These findings were validated in the TCGA cohort (BIRC5: coeff (median) $0.222(0.070$ $0.273), \quad p$ value (median) $6.1 \times 10^{-10} \quad(0.042-5.6 \times$ $\left.10^{-16}\right)$; RGN (female): coeff -0.409 to $-0.375, p$ value $4.15 \times 10^{-15}-9.14 \times 10^{-13}$; RGN (male): coeff -0.573 to $-0.507, p$ value $\left.1.63 \times 10^{-43}-7.04 \times 10^{-33}\right)$. For $R G N$, we show a clear negative correlation of gene transcription and methylation in the promoter site, which points towards an aberrant methylation in NSCLC, exemplarily visualized in Fig. 1e. While no significant differences in BIRC5 expression between adenocarcinomas and squamous cell carcinomas have been detected $(p=0.53, t$ test), adenocarcinomas showed by trend a slightly higher RGN RNA level than squamous cell carcinomas $(p=0.053, t$ test $)$ (Fig. 1b). This finding is supported by analyzing gene expression data of a larger lung cancer dataset provided by the TCGA consortium $\left(p<2.2 \times 10^{-16}, t\right.$ test). The hypermethylation of BIRC5 taking place mainly in the 3 '-UTR region showing no differences at the promoter region suggests a secondary regulation, e.g., by a RNA or LncRNA. The methylation differences of BIRC5 therefore seem to be non-aberrant and dependent possibly on the cell of origin, which is reflected in the separation of adenocarcinomas and squamous cell carcinomas by analyzing the methylation of BIRC5 (Fig. 1a).

Kaplan-Meier analyses were performed in a separate collective of patients from data generated using Affymetrix chips and retrieved from kmplot.com (Fig. 1c) [8]. A high expression of BIRC5 on the RNA level was negatively correlated with the survival of patients in adenocarcinomas (Fig. 1c (I)) while a high $R G N$ expression was positively correlated with survival (Fig. 1c (III)). No such correlation was found in squamous cell carcinomas (Fig. 1c (II and IV)). This separation was even more distinct for both genes in stage 1 adenocarcinomas (Fig. 1c ( $\mathrm{V}$ and VII)). Further inclusion of smoking habits revealed that smokers with low expression of BIRC5 show a better outcome in stage 1 tumors (Fig. 1c (VI)), while high expression of $R G N$ in stage 1 adenocarcinomas seems to be protective in never smokers (Fig. 1c (VIII)). Furthermore, we found similar data on the expression level differences between tumors and tumor-free tissues also in kmplot.com $(N=2437$ tumors, 86 tumor-free. BIRC5: median tumors $=244$, median tumor-free $=149[N=86]$; RGN: median tumors $=192$, median tumor-free $=383$ ).

On the protein level, we observed an elevated expression of BIRC5 (Fig. 1d) in lung tumors, regardless of the histological sub-entity (adenocarcinoma/squamous cell carcinoma). Moreover, we frequently observed high levels of BIRC5 expression in tumor-adjacent immune cells (29/40 cases).

Interestingly, we found similar expression levels for RGN in the tumor and tumor-free areas, which are below the levels of BIRC5, suggesting regulation on the translational level or protein turnover (Fig. 1d).

In summary, we show that BIRC5 and RGN are epigenetically modified in human NSCLC tissues compared 


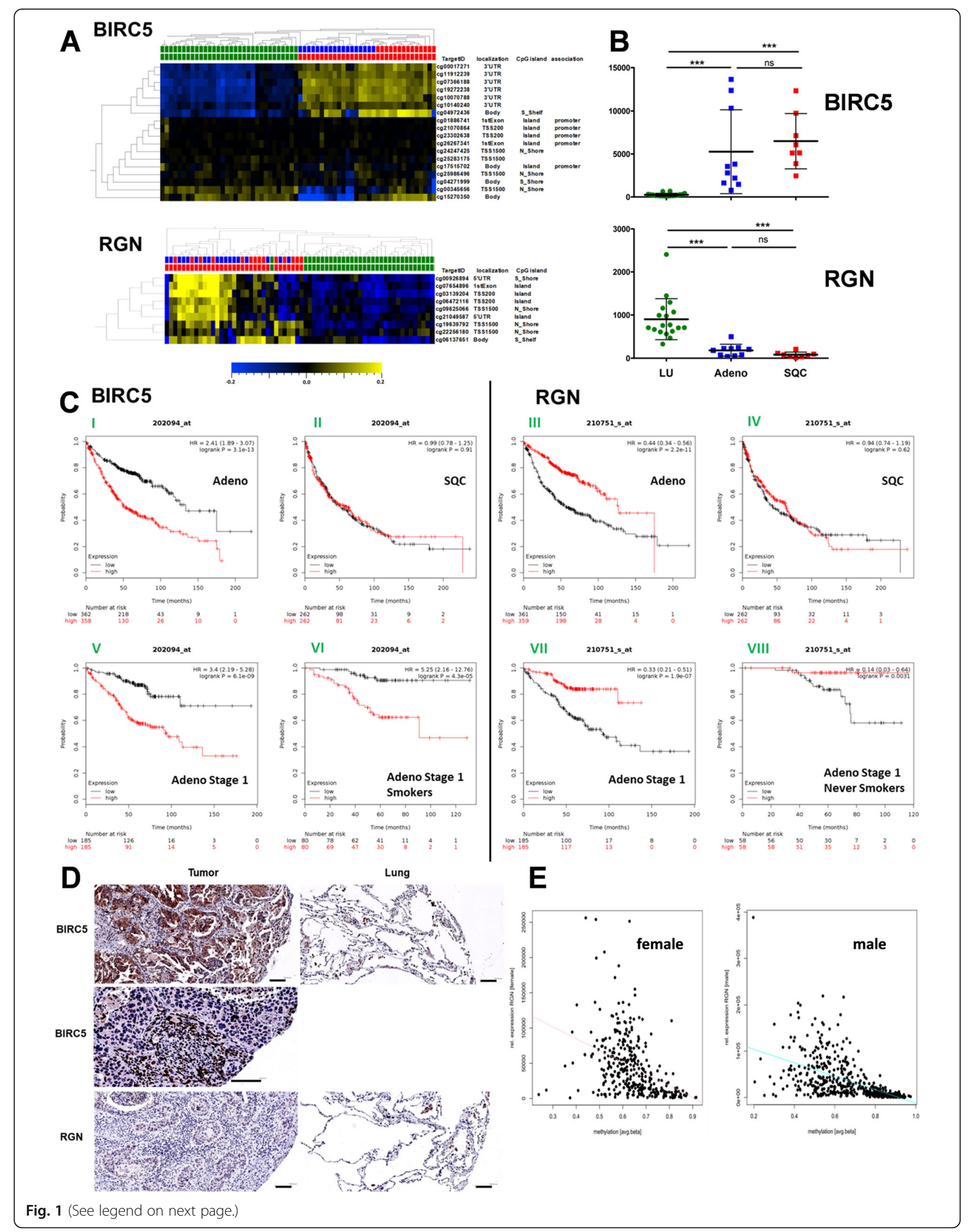


(See figure on previous page.)

Fig. 1 BIRC5 and RGN are differentially methylated and expressed in NSCLC and tumor-free lung tissues. a Normalized methylation analyses of CpG loci on Human Methylation 450k BeadChip (upper top bar: green — controls, blue—adenocarcinomas, red_squamous cell carcinomas; lower top bar: green - controls, red - tumor samples; heatmap: yellow high, blue low DNA methylation values; mean DNA methylation = 0 ; the target IDs of the $\mathrm{CpG}$ loci, their localization, and functional association are indicated). b Relative gene expression level of BIRC5 and RGN as quantile-normalized expression values of tumor-free lung tissues and matched tumors depicting the mean in the $99.9 \%$ confidence interval with error bars (green: controls, blue: adenocarcinomas, red: squamous cell carcinomas). One-way ANOVA and Tukey's multiple comparison test were used with $p \leq 0.001\left(^{* * *}\right)$ regarded significant. c Survival analysis using RNA expression data retrieved from kmplot.com selecting Affymetrix-ID 202094 for BIRC5 and 210751_s for RGN. d Exemplary results from IHC analyses displaying expression of BIRC5 (upper and middle panel) and RGN (lower panel) on the protein level in tumors (left) and tumor-free lung tissues (right) from the same donors (scale bars $=100 \mu \mathrm{m}$ ). Middle panel shows the expression of BIRC5 in tumor-adjacent immune cells. e Correlation of DNA methylation (cg06472116, located on chr. X) vs. gene expression (RGN) in female [left graph] (Pearson's correlation coef $-0.4127 ; p$ value $<1.1550 \times 10^{-15}$ ) and male [right graph] donors (Pearson's correlation coef $-0.5682 ; p$ value $<1.3136 \times 10^{-44}$ )

to tumor-free tissues, resulting in increased $(B I R C 5)$ or decreased $(R G N)$ transcription with a strong increase of BIRC5, but not a decrease of RGN on the protein level. We show correlations of both antithetic molecules with the survival of adenocarcinoma patients, especially in stage 1 and depending on smoking habits, which, although deserving further validation, might be useful in prognostic approaches.

\section{Abbreviations}

BIRC5: Survivin; IHC: Immunohistochemistry; NSCLC: Non-small cell lung cancer; RGN: Regucalcin; TSS: Transcription start site

\section{Acknowledgements}

The authors thank Jasmin Tiebach, Maria Lammers, and Christian Rosero for the excellent technical assistance.

\section{Authors' contributions}

TG conceived of the study. DN, SM, SAK, OA, and TG prepared and analyzed the data. MR, ChK, and KFR edited the manuscript. SM, DN, OA, and TG wrote the manuscript. All authors read and approved the final manuscript.

\section{Funding}

This work was funded by the German Center for Lung Research (DZL; 82DZL001A5). Patient tissues were provided by the BioMaterialBank North, which is funded in part by the Airway Research Center North (ARCN), member of the German Center for Lung Research (DZL), and is member of popgen 2.0 network (P2N), which is supported by a grant from the German Ministry for Education and Research (01EY1103).

\section{Availability of data and materials}

The datasets used and/or analyzed during the current study are available from the corresponding author on reasonable request.

\section{Ethics approval and consent to participate}

The use of patient material was approved by the local ethics council at the University of Lübeck (AZ-12-220).

\section{Consent for publication}

Not applicable.

\section{Competing interests}

The authors declare that they have no competing interests.

\section{Author details}

${ }^{1}$ Pathology of the University Medical Center Schleswig-Holstein, Campus Lübeck and the Research Center Borstel, Leibniz Lung Center, Borstel, Germany. 'LungenClinic Großhansdorf, Großhansdorf, Germany. ${ }^{3}$ Airway Research Center North (ARCN), Member of the German Center for Lung Research (DZL), Großhansdorf, Germany. ${ }^{4}$ Institute of Human Genetics, Christian-Albrechts-University Kiel and University Medical Center Schleswig-Holstein, Campus Kiel, Kiel, Germany. ${ }^{5}$ Institute of Human Genetics, UIm University and University Medical Center UIm, Ulm, Germany.
Received: 24 June 2019 Accepted: 22 October 2019

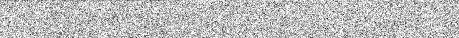

\section{References}

1. Campisi J. Aging, cellular senescence, and cancer. Annu Rev Physiol. 2013; 75:685-705

2. Marwitz S, Heinbockel L, Scheufele S, Kugler C, Reck M, Rabe KF, et al. Fountain of youth for squamous cell carcinomas? On the epigenetic age of non-small cell lung cancer and corresponding tumor-free lung tissues. Int J Cancer. 2018:143(12):3061-70.

3. Lyu H, Huang J, He Z, Liu B. Epigenetic mechanism of survivin dysregulation in human cancer. Sci China Life Sci. 2018;61(7):808-14.

4. Cui X, Shen D, Kong C, Zhang Z, Zeng Y, Lin X, et al. NF-kB suppresses apoptosis and promotes bladder cancer cell proliferation by upregulating survivin expression in vitro and in vivo. Sci Rep. 2017;7:40723.

5. Yamaguchi M, Osuka S, Shoji M, Weitzmann MN, Murata T. Survival of lung cancer patients is prolonged with higher regucalcin gene expression: suppressed proliferation of lung adenocarcinoma A549 cells in vitro. Mol Cell Biochem. 2017:430(1-2):37-46.

6. Marwitz S, Scheufele S, Perner S, Reck M, Ammerpohl O, Goldmann T. Epigenetic modifications of the immune-checkpoint genes CTLA4 and PDCD1 in non-small cell lung cancer results in increased expression. Clin Epigenetics. 2017;9:51.

7. Kreck B, Richter J, Ammerpohl O, Barann M, Esser D, Petersen BS, Vater I, Murga Penas EM, Bormann Chung CA, Seisenberger S, Lee Boyd V, Smallwood S, Drexler HG, Macleod RA, Hummel M, Krueger F, Häsler R, Schreiber S, Rosenstiel P, Franke A, Siebert R. Base-pair resolution DNA methylome of the EBV-positive endemic Burkitt lymphoma cell line DAUDI determined by SOLiD bisulfite-sequencing. Leukemia. 2013;27(8):1751-3.

8. Nagy A, Lánczky A, Menyhárt O, Győrffy B. Validation of miRNA prognostic power in hepatocellular carcinoma using expression data of independent datasets. Sci Rep. 2018:8:9227.

\section{Publisher's Note}

Springer Nature remains neutral with regard to jurisdictional claims in published maps and institutional affiliations.

\section{Ready to submit your research? Choose BMC and benefit from:}

- fast, convenient online submission

- thorough peer review by experienced researchers in your field

- rapid publication on acceptance

- support for research data, including large and complex data types

- gold Open Access which fosters wider collaboration and increased citations

- maximum visibility for your research: over $100 \mathrm{M}$ website views per year

At $\mathrm{BMC}$, research is always in progress.

Learn more biomedcentral.com/submission 